\title{
Physical Diagnosis in the Contemporary Era
}

There is a consensus that clinical evaluation gives $80 \%$ of diagnoses. This is based on consistent historical experience. We already know that the positive value of signs, symptoms or diagnostic tests increases as the accuracy of clinical evaluation improves. Nowadays, as concepts of accuracy, sensitivity, specificity and predictive values are becoming popular in medical practice, the accuracy of the semiology needs to be reassessed in the light of such concepts. What are the characteristics of a physical heart examination when compared with echodoppler cardiography? What is the sensitivity and specificity of physical spleen examination when compared with ultrasonography, in which the CT scan is considered the gold standard? The Federal Council for Internal Medicine of the American College of Physicians moves a step forward in their guide to curriculum development, with the following:

\section{"Physical Diagnosis}

Even in this era of burgeoning diagnostic technology, the physical examination remains among the internist's most accurate set of tools. These skills play an essential role in estimating the pre-test probability of disease, which is the starting point for test interpretation. Moreover, there is an increasing body of knowledge about accuracy of physical signs. In many instances, bedside assessment is superior to noninvasive technology; in almost all cases, it is more accessible and cost-effective.

Expertise in physical diagnosis is a valued characteristic of the well-trained internist. Instruction in and evaluation of physical diagnosis skill should be part of every residency program's curriculum. We present first a list of general competency objectives for physical diagnosis, followed by an organ-specific list of the physical

* $M D, P h D, M C E$

Chaiman, Department of Internal Medicine, Escola

Paulista de Medicina, Editor, São Paulo Medical Journal. findings and maneuvers with which all residents should be familiar.

\section{Competencies for Physical Diagnosis}

- Understand how to apply the concept of operating characteristics (specificity, sensitivity, and likelihood ratios) to the interpretation of physical examination findings

- Understand the pathophysiologic explanation for common physical findings

- Know when to abandon a physical finding because new evidence has impugned its validity and when to adopt new findings that have been shown to be clinically useful

- Examine patients efficiently and systematically, maximizing accuracy and completeness, ensuring that the patient is comfortable, and protecting the patient's modesty

- Use the physical examination in the context of the entire clinical database to evaluate the patient efficiently and effectively

- Know the content of the screening physical examination that is appropriate for each patient's age, sex, and particular risk factors

- Utilize repeated, focused physical examinations to follow the course of a patient's illness

- Use physical findings to make decisions in settings that do not allow for extensive diagnostic testing."

\section{REFERENCE}

Federated Council for Internal Medicine, Task Force on the Internal Medicine Residency Curriculum. Graduate education in internal medicine: a resource guide to curriculum development. Philadelphia: American College of Physicians; 1997. ISBN 0943126-60-6 